\title{
A GIANT MERIDIAN CIRCLE - REFLECTOR
}

\author{
V.N.YERSHOV \\ Pulkovo Observatory, \\ 196140 St.Petersburg, Russia \\ e-mail: yersh@gao.spb.su
}

A $1.5 \mathrm{~m}$ reflector is proposed for infrared and optical meridian observations in order to extend the fundamental coordinate system to faintest objects and to the K-infrared waveband. Classical meridian circles are unfit for the infrared observations because their lens objectives do not give good images in the infrared. But reflectors are almost never used as meridian circles due to uncertainties in their optical axis position. The main problem is that the secondary mirror is not connected with the micrometer and the circle reading system. In order to overcome this difficulty the author proposes to use an intermediary focal plane between the primary and the secondary mirrors where a luminous reference grid of wires might be placed. The Gregory optical scheme has such a focal plane, and its secondary mirror forms images of a star and the grid at the micrometer's detecting area. At the same time a special champher around the primary's central hole forms anautocollimated image of the grid near the grid itself. The micrometer measures the star image coordinates relative to two images of the reference grid. So, observations will not be affected by displacements of the secondary mirror and by those of the micrometer. The telescope's equivalent focal length has been chosen as $3 m$, and the optical system has been transformed into an aplanatic Mersenne combined with an aplanatic focal reducer corrector (Popov, 1988). A new autocollimated circle reading system is chosen for the instrument (Yershov and Nemiro, 1994). The observations will be linked to the fixed optical axis of two long-focus collimators placed at the prime vertical plane.

\section{References}

Popov, G.M. (1988), Modern Astron. Optics, "Science", Moscow, 146-147.

Yershov, V.N., Nemiro A.A. (1994), An Autocollimation Circle Reading System for the Infrared Merid. Instr., IAU Symp.166, The Haague, 1994 